\title{
First live records of the ruby seadragon (Phyllopteryx dewysea, Syngnathidae)
}

\author{
Greg W. Rouse ${ }^{1 *} \mathbb{D}$, Josefin Stiller ${ }^{1}$ and Nerida G. Wilson ${ }^{1,2,3}$
}

\begin{abstract}
Until recently, only two species of seadragon were known, Phycodurus eques (the leafy seadragon) and Phyllopteryx taeniolatus (the common seadragon), both from Australia. In 2015, we described a new species of seadragon, Phyllopteryx dewysea (the ruby seadragon). Although the leafy and common seadragons are well known and commonly seen in aquarium exhibits world-wide, the ruby seadragon was known only from four preserved specimens, leaving many aspects of its biology unknown. Based on specimen records, it was speculated that the ruby seadragon normally lives at depths beyond recreational SCUBA diving limits, which may also explain why it went undiscovered for so long. The ruby seadragon also bears a superficial resemblance to the common seadragon, with a number of specimens misidentified in museum collections. The only recent live-collected specimen was trawled from the Recherche Archipelago, a cluster of over 100 islands in Western Australia. We took a small remotely operated vehicle (miniROV) to this locality to obtain the first images of live ruby seadragons. We made observations on the seadragon habitat and behavior, including feeding. We also provide new key observations on their morphology, notably that they lack dermal appendages and have a prehensile tail. We recommend that the ruby seadragon be protected as soon as practicable.
\end{abstract}

Keywords: Australia, Biodiversity, Marine, Syngnathid, Seadragon

\section{Background}

The original description of the ruby seadragon (Phyllopteryx dewysea, Stiller, Wilson and Rouse 2015) was based on four specimens, three from near Perth in Western Australia. One of these had been collected in 1919 as a beach-washed specimen and the other two were trawled from $72 \mathrm{~m}$ in 1956. The fourth specimen (holotype) was a male carrying a brood of eggs, which was trawled in 2007 from $\sim 50 \mathrm{~m}$ depth in the Recherche Archipelago, Western Australia. This locality is than more than $1000 \mathrm{~km}$ to the east of where the older specimens had been collected. The holotype and brood were preserved in ethanol, allowing for DNA sequencing. As well as marked DNA evidence for the ruby seadragon as a new species, there were clear diagnostic morphological features, including its red color, 18 trunk rings, forward-pointing dorsal spines on the 11th trunk ring, and an enlarged pectoral area. Dermal appendages are seen in the common and leafy seadragons and function as camouflage in the temperate algal reef habitats

\footnotetext{
* Correspondence: grouse@ucsd.edu

'Scripps Institution of Oceanography, UCSD, La Jolla, CA 92037, USA

Full list of author information is available at the end of the article
}

(Kuiter 2000). Although the museum specimens of ruby seadragons possessed the enlarged bony spines to which these appendages attach in the other species, the appendages themselves were absent. It was not clear if the appendages had been lost postmortem. Other outstanding questions included aspects of their behavior and what kind of habitat they occupied. To obtain the first live observations of the ruby seadragon, we went to the Recherche Archipelago in Western Australia in early April 2016.

\section{Materials and methods}

The holotype of $P$. dewysea had been collected on a biodiversity survey and trawl coordinates were available. Collection details for the other known specimens were less detailed, and these were collected many years ago without any subsequent records despite neighbouring an urban environment (Stiller et al. 2015). We therefore targeted the Recherche Archipelago as the best locality to find living ruby seadragons. This idea was reinforced by the fact that after the ruby seadragon was described, another specimen washed ashore in February, 2015 at Culver Cliffs, east of the Recherche 
Archipelago (Additional file 1) (Della Vedova 2015) and another washed up near Esperance, and was donated to the Western Australian Museum (WAMP34456.001X). As the 50+ meters depth made SCUBA diving activities impractical, we deployed a Teledyne SeaBotix vLBV300 (Anonymous 2015) remotely operated vehicle (miniROV) with a low-light video camera to make observations. As the boat charter was for a restricted period and inclement weather prevented most operations, we only had a single day (April 7) at the site close to the type locality. We undertook four dives of up to 1-h duration on this day. Plans to attempt to catch ruby seadragons using the miniROV were abandoned owing to the sea conditions.

\section{Results}

The ocean swell had a marked influence at the 50+ meters depth at which the miniROV was operating (Additional file 2: Movie S1). The habitat was mostly deeply-rippled sand over a hard reef substrate at a depth of more than $50 \mathrm{~m}$ and a bottom temperature of $18^{\circ}$ Celsius. Numerous large demosponges, gorgonians and hydroid cnidarians, erect fenestrate and foliose bryozoan colonies, and algae (including Caulerpa cf. longifolia) were present. On the second dive, at 54 $m$ depth, we observed a ruby seadragon near a sponge and proceeded to follow the fish. This specimen (Fig. 1a), sex indeterminate, had no dermal appendages and had a curled, likely prehensile tail with a yellow tip (as seen in the beach-washed specimen, Additional file 1). The fish tended to move slowly away from the miniROV, turning backwards and forwards to hold position in the surge, but when it encountered a large object such as an erect sponge, it would often linger before moving on (Additional file 2: Movie S1). After $8 \mathrm{~min}$, we encountered a second ruby seadragon (Fig. 1b), possibly female because of the deeper and more circular body shape, with a notably darker red color and more obvious vertical bars. The two fish stayed in the same vicinity for about 1 min (Additional file 2: Movie S1). We then followed the second fish for $20 \mathrm{~min}$. This fish also lacked dermal appendages and also had a curled tail (Fig. 1c). We did not directly observe either fish hold onto an object with their tail, but they regularly trailed the tail over an object, in a manner which resulted in a mild recoil from the object as the fish departed (Additional file 2: Movie S1). During nearly $30 \mathrm{~min}$ of observations on the two ruby seadragons we saw 10 feeding strikes, mostly near the bottom, but in some cases the fish rose slightly above the benthos to strike (Additional file 2: Movie S1). The other three dives that day in the same locality (a further $100 \mathrm{~min}$ of observation) revealed no other ruby seadragons.
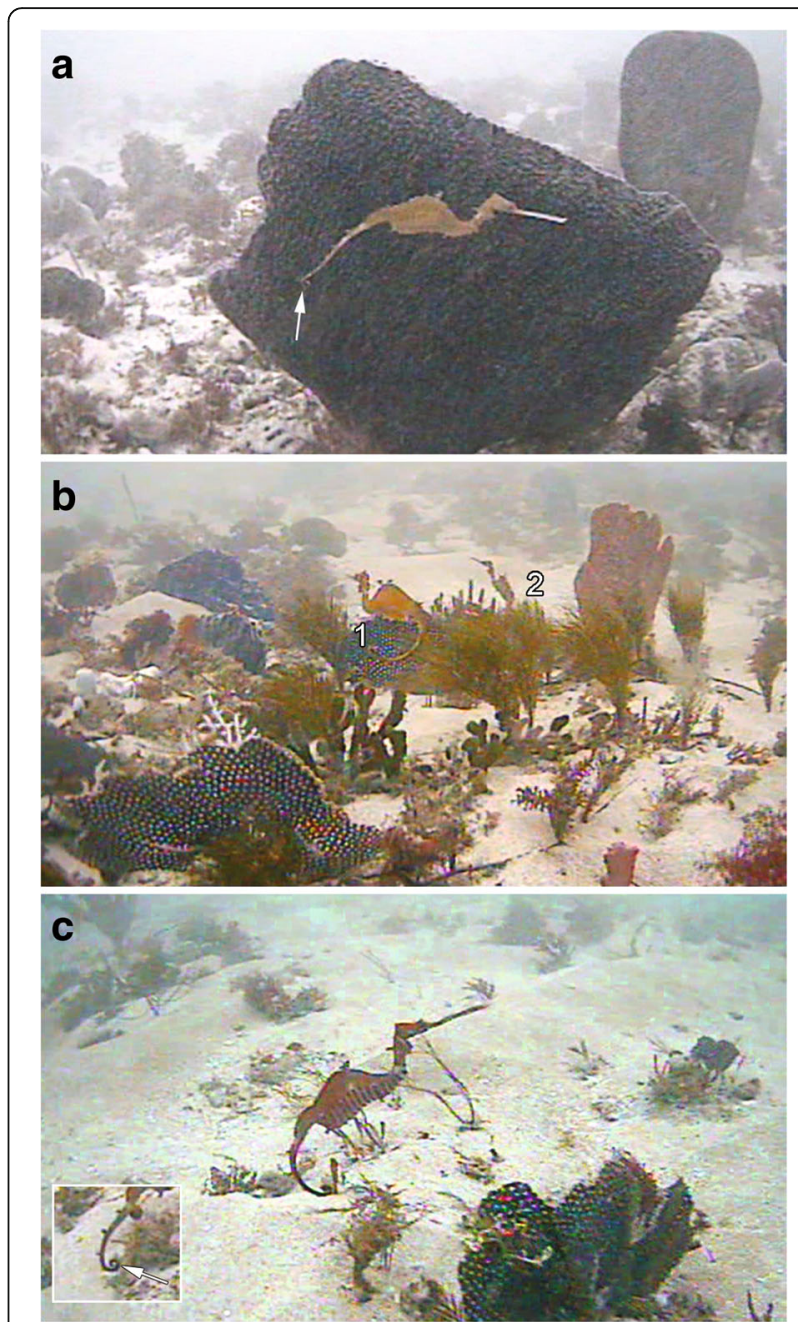

Fig. 1 The first live observations of the ruby seadragon, (Phyllopteryx dewysea), in the Recherche Archipelago, Western Australia. a The first specimen set against a large sponge, showing the absence of dermal appendages. Arrow points to yellow-tipped tail that is uncoiled. $\mathbf{b}$ Both individuals in the typical habitat. $\mathbf{c}$ The second specimen showing the coiling of the tail. Note the lack of dermal appendages. The inset shows a detail of the apparently prehensile tail

\section{Discussion}

The documentation of living ruby seadragons at more than $50 \mathrm{~m}$ depth confirms that these fish live at deeper depths than leafy and common seadragons and in a very different habitat. Leafy seadragons occur along the coast of Western and South Australia from south of Perth to east of Adelaide and are generally found in depths from 3 to $25 \mathrm{~m}$ near brown algae, seagrass, and sand (Kuiter 2000; Stiller et al. 2017; Connolly et al. 2002a). Leafy seadragons are known from as deep as $30 \mathrm{~m}$ and with a reddish coloration (Kuiter 2000). Ultrasonic tracking of leafy seadragons showed that they stay over Posidonia seagrass, macroalgae-covered reefs and sand, but seemed to avoid Amphibolis seagrass and boulders with 
brown algae (Connolly et al. 2002b). Common seadragons have wider geographic range, extending east to the central New South Wales coast and also around Tasmania (Wilson et al. 2016). They show a similar depth preference to leafy seadragons and are also known for living associated with rocky reefs, sand patches, kelp and seagrass (Kuiter 2000; Sanchez-Camara and Booth 2004). The video results for the ruby seadragon clearly show that these fish live in a very different habitat compared to their relatives. They also lack dermal appendages as opposed to the prominent appendages of leafy and common seadragons. In the sparse habitat they occupy, appendages would serve little purpose as camouflaging agents and could add significant costs in drag or fluid resistance, particularly in strong surge. It appears that at these low-light depths, an efficient camouflage strategy for ruby seadragons is to rely on cryptic red coloration.

Surprisingly, we also saw that the ruby seadragon has what appears to be a prehensile tail. Although neither fish was observed to use it in a direct hold, the conditions on the day were relatively calm. The fish would often be exposed to much stronger surge, and then may well use their tails to stop from being swept off the very limited reefal habitats. A prehensile tail is found across a range of Syngnathidae and appears to have evolved at least five times from an ancestral state where the tail possessed a tail fin (Neutens et al. 2014). With regards to seadragons, the phylogeny and transformation for tail shown by Neutens et al. (2014) shows the leafy and common seadragon as a clade tail lacking a tail fin and without grasping capacities. This condition was reasonably interpreted as a single loss of prehensile ability by Neutens et al. (2014) since pipehorses (Solegnathus and Syngnathoides) have prehensile tails and are the closest relatives of the seadragon clade. In these pipehorses, the ability to curl the tail appears to be facilitated by a reduction of the plates, either ventrally (Solegnathus) or entirely (Syngnathoides) (Neutens et al. 2014). The ruby seadragon holotype (Stiller et al. 2015) lacks the very tip of the tail $(\mathrm{ca} .1 \mathrm{~cm})$ and so the available microCT scan cannot detect this. Nonetheless, the plates do not appear to be reduced as in pipehorses and all rows seem to be present along the tail in the ruby seadragon.

The discovery that the ruby seadragon has a prehensile tail complicates the scenario of the evolution of prehensile tails in this group, as it is the closest relative to the common seadragon (Stiller et al. 2015), which cannot bend its tail. One parsimonious explanation is that the absence of a prehensile tail in the leafy and common seadragons has independently evolved in each species and that the prehensile tail of the ruby seadragon is a retained plesiomorphic condition. Alternatively, the prehensile tail may have been lost in an ancestor of all seadragons, and the ruby seadragon has re-acquired a prehensile tail. To help choose between these scenarios, detailed observations of leafy and common seadragon tails could be compared to the available microCT scan of the ruby seadragon (Stiller et al. 2015).

\section{Conclusions}

Our observations on living ruby seadragons, as well the specimens either trawled (Stiller et al. 2015) or washed ashore (Della Vedova 2015), including a male with a brood of young, suggest there is a viable population in the vicinity of the Recherche Archipelago. The earlier records from further west (Stiller et al. 2015) suggest the ruby seadragon may have a widespread distribution in Western Australia, though there is little contemporary evidence to support this. As with the leafy and common seadragons we encourage the protection of the ruby seadragon as soon as practicable. We are proceeding with actions to nominate the ruby seadragon for listing at state and federal levels, to afford it the protection already available to the other two seadragon species.

\section{Additional files}

Additional file 1: A ruby seadragon, Phyllopteryx dewysea that washed up on the Point Culver cliffs in Western Australia. Photos taken by Zoe Della Vedova. A. The specimen appeared to be fresh and still showed the bright red coloration and light snout markings. B. Although the curling of the tail seems to disappear after death, a yellow tip of the tail was visible (arrow). (JPG $2366 \mathrm{~kb}$ )

Additional file 2: Movie S1. Underwater footage of living ruby seadragons, Phyllopteryx demysea illustrating their typical habitat and behavior. The video was taken using a miniROV at ca. 54 m depth in Recherche Archipelago, Western Australia. (M4V 16283 kb)

\section{Acknowledgements}

We thank Dewy White (Lowe Family Foundation) for funding this expedition. We thank Total Marine Technology (TMT), especially Chris Porter, for their technical support, supplying the miniROV and pilot Daffyd Philips for expert technique. We also thank Colin Hampson and Kinglsey Whitta (crew of the Southern Conquest) and Peter and Jaimen Hudson of Esperance Diving and Fishing. Zoe Della Vedova is thanked for the photos of the beach-cast specimen. We thank two anonymous reviewers for their valuable comments on the manuscript. We have no competing interests.

\section{Funding \\ Lowe Family Foundation.}

Availability of data and materials

Electronic supplementary material is available at http:

Authors' contributions

GWR, JS, NGW conceived the project, collected field data and drafted the manuscript. All authors gave final approval for publication.

Competing interests

The authors declare that they have no competing interests.

Consent for publication

Not applicable.

Ethics approval

Approval to collect ruby seadragons was issued under the Western

Australian Fish Resources Management Act 1994, Exemption number 2725. 
We also had University of California, San Diego Institutional Animal Care and Use Committee approval under Protocol S02118. We did not attempt to collect the seadragons owing to the sea conditions and took video only.

\section{Author details}

'Scripps Institution of Oceanography, UCSD, La Jolla, CA 92037, USA.

${ }^{2}$ Western Australian Museum, Locked Bag 49, Welshpool DC, WA 6986,

Australia. ${ }^{3}$ University of Western Australia, Crawley, WA 6009, Australia.

Received: 17 June 2016 Accepted: 28 November 2016

Published online: 13 January 2017

\section{References}

Anonymous: vLBV300. http://www.teledynemarine.com/lbv300-5/?BrandID=19. Accessed 5 Dec 2016.

Connolly RM, Melville AJ, Preston KM. Patterns of movement and habitat use by leafy seadragons tracked ultrasonically. J Fish Biol. 2002a;61:684-95.

Connolly RM, Melville AJ, Keesing JK. Abundance, movement and individual identification of leafy seadragons Phycodurus eques (Pisces: Syngnathidae). Mar Freshwater Res. 2002b:53:777-80.

Della Vedova Z. Rare ruby seadragon washes up on WA cliffs. http://www. australiangeographic.com.au/blogs/ag-blog/2015/03/rare-ruby-seadragonwashes-up-on-wa-cliffs. Accessed 30 May 2016.

Kuiter RH. Seahorses, pipefishes and their relatives - a comprehensive guide to syngnathiformes. Chorleywood: TMC Publishing; 2000.

Neutens C, Adriaens D, Christiaens J, De Kegel B, Dierick M, Boistel R, Van Hoorebeke L. Grasping convergent evolution in syngnathids: a unique tale of tails. J Anat. 2014;224:710-23.

Sanchez-Camara J, Booth DJ. Movement, home range and site fidelity of the weedy seadragon Phyllopteryx taeniolatus (Teleostei: Syngnathidae). Environ Biol Fishes. 2004;70:31-41.

Stiller J, Wilson NG, Rouse GW. A spectacular new species of seadragon (Syngnathidae). Royal Society Open Science. 2015;2:140458.

Stiller J, Wilson NG, Donnellan SC, Rouse GW. The leafy seadragon, Phycodurus eques, a flagship species with low but structured genetic variability. J Hered. 2017. doi:10.1093/jhered/esw1075.

Wilson NG, Stiller J, Rouse GW. Barriers to gene flow in common seadragons (Syngnathidae: Phyllopteryx taeniolatus). Conserv Genet. 2016. doi:10.1007/ s10592-10016-10881-y.

\section{Submit your next manuscript to BioMed Central} and we will help you at every step:

- We accept pre-submission inquiries

- Our selector tool helps you to find the most relevant journal

- We provide round the clock customer support

- Convenient online submission

- Thorough peer review

- Inclusion in PubMed and all major indexing services

- Maximum visibility for your research

Submit your manuscript at www biomedcentral.com/submit
() BioMed Central 